\title{
High Resolution Observations of Interface Dynamics Using a Direct Electron Detection Camera
}

\author{
T. Radetic ${ }^{1}$, A. Gautam ${ }^{1}$, C. Ophus ${ }^{1,}$ C. Czarnik ${ }^{2}$ and U. Dahmen ${ }^{1}$ \\ ${ }^{1}$ National Center for Electron Microscopy, LBNL, Berkeley California 94720 USA \\ ${ }^{2}$ Gatan Inc., Pleasanton, California 94588 USA
}

Atomic-scale mechanisms of interface motion are of key interest for processes such as grain growth, deformation and phase transformations. The direct observation of such mechanisms by transmission electron microscopy is of great importance for our understanding and predictive modeling of the behavior of new materials. Recent advances in instrumentation have made it possible to extend the range of spatial and temporal resolution of such observations [1].

In this work, we report on observations of the motion of internal interfaces during phase transformations at elevated temperature using a Gatan K2-IS direct electron detection camera installed on an uncorrected FEI Titan transmission electron microscope. Our observations record the motion of singular interfaces such as grain boundaries in $\mathrm{Au}$ and interface boundaries between liquid $\mathrm{Pb}$ and solid $\mathrm{Al}$ at temperatures up to $400^{\circ} \mathrm{C}$ at lattice resolution.

The migration of singular interfaces typically involves nucleation and propagation of steps. Observations by Merkle et al. [2] by high resolution microscopy showed grain boundaries in Au and Al to move by glide, step motion and cooperative shuffles. Step motion was the most prominent mode for singular boundaries at a deep energy cusp. Figure 1 illustrates the motion of a step in a singular high-angle tilt grain boundary in $\mathrm{Au}$ at a temperature of $217^{\circ} \mathrm{C}$. The two grains are misoriented relative to each other by $90^{\circ}$ around the $<110>$ axis, which coincides with the beam direction. Along the boundary, (110) planes of the upper grain lie opposite (001) planes of the lower grain. A step, two atomic-planes high, is seen to move laterally during six sequential frames recorded at 400 frames per second. At standard video rates, these six frames would be recorded as a single averaged image, which would leave the motion of the step unresolved. At the rate of imaging illustrated here, it becomes possible to track the random motion of boundaries and extract their mobilities [3]. This is of particular importance for the grain boundary observed in this work because this boundary controls the rate of interface motion during capillary shrinkage of island grains [4].

Figure 2 shows dynamic observations of the interface between liquid $\mathrm{Pb}$ and solid $\mathrm{Al}$ at a temperature of $400^{\circ} \mathrm{C}$. All images were recorded at lattice resolution, allowing precise measurement of the shape (b) and position ( $\mathrm{c}-\mathrm{e}$ ) of the liquid $\mathrm{Pb}$ inclusion as the undergo Brownian motion at elevated temperature. The increased resolution compared to previous video-rate data greatly improves the accuracy of measurement and thus helps distinguish between competing mechanism of motion $[5,6]$.

\section{References:}

[1] Dahmen U et al. P. Philos T R Soc A 2009;367:3795.

[2] Merkle K, Thompson L, Phillipp F. Interface Sci 2004;12:277.

[3] Trautt ZT, Upmanyu M, Karma A. Science 2006;314:632.

[4] Radetic T, Ophus C, Olmsted DL, Asta M, Dahmen U. Acta Materialia 2012;60:7051.

[5] Johnson E, Prokofjev S, Zhilin V, Dahmen U. Z Metallkd 2005;96:1171. 
[6] The National Center for Electron Microscopy at LBNL is supported by the U.S. Department of Energy under Contract \# DE-AC02-05CH11231

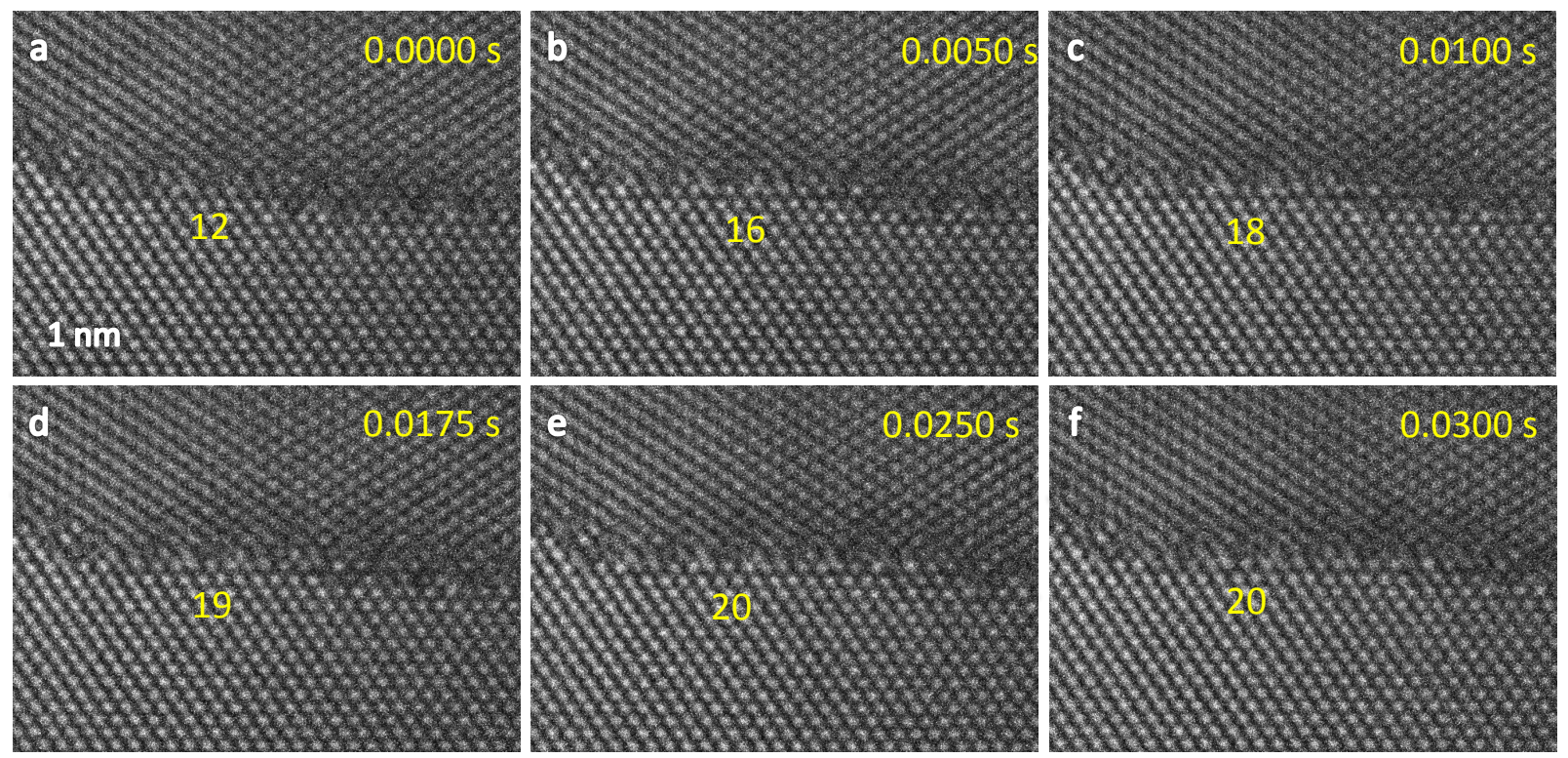

Figure 1 Sequence of images showing propagation of a step (2 atomic planes high) recorded with K2 camera at $217^{\circ} \mathrm{C}$. Step position marked as the number of plane spacings from 12 to 20 . The whole sequence (a-f) corresponds to a single frame at regular TV rate. Over a more extended time frame this step moves back and forth randomly.
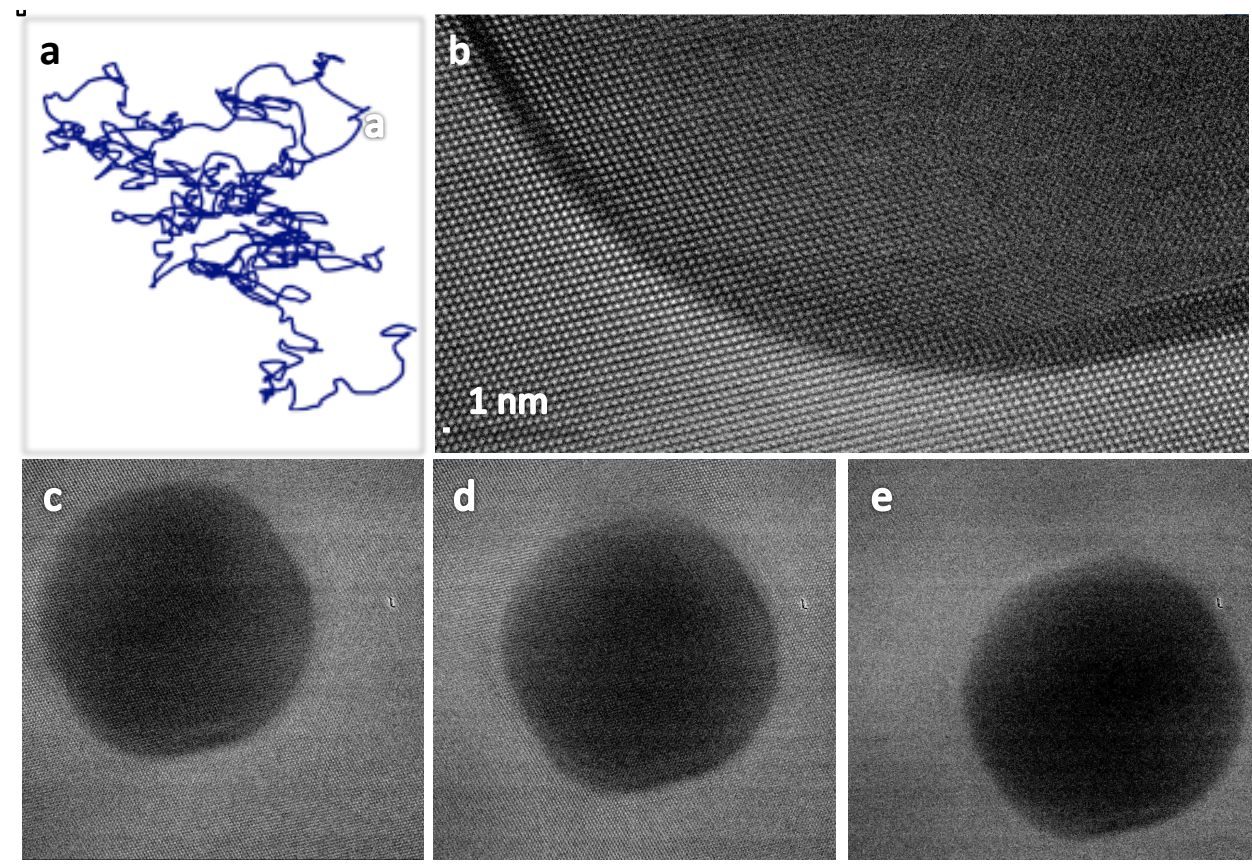

Figure 2 a) Trajectory tracing random walk of liquid $\mathrm{Pb}$ inclusions in solid $\mathrm{Al}$; (b-e) image sequence recorded at $400^{\circ} \mathrm{C}$ and $400 \mathrm{fps}$ maintaining lattice resolution in the Al matrix while showing the projected inclusion shape accurately (b), allowing precise measurement of shape and position (c-e). 